OPEN ACCESS

Edited by: Maria Fragoso,

Institute of Cancer of Sao Paulo, Brazil

Reviewed by:

Antonio Lerario,

University of Michigan, United States

Erick J.R. Silva,

São Paulo State University, Brazi

*Correspondence:

Andre Lacroix

andre.lacroix@umontreal.ca

Specialty section:

This article was submitted to

Cellular Endocrinology,

a section of the journal

Frontiers in Endocrinology

Received: 04 June 2021

Accepted: 09 August 2021

Published: 27 August 2021

Citation

Younes N, Bourdeau I and Lacroix A (2021) Latent Adrenal Insufficiency: From Concept to Diagnosis.

Front. Endocrinol. 12:720769. doi: 10.3389/fendo.2021.720769

\section{Latent Adrenal Insufficiency: From Concept to Diagnosis}

\author{
Nada Younes, Isabelle Bourdeau and Andre Lacroix* \\ Division of Endocrinology, Department of Medicine and Research Center, Centre Hospitalier de l'Université de Montréal \\ (CHUM), Montréal, QC, Canada
}

Primary adrenal insufficiency (PAl) is a rare disease and potentially fatal if unrecognized. It is characterized by destruction of the adrenal cortex, most frequently of autoimmune origin, resulting in glucocorticoid, mineralocorticoid, and adrenal androgen deficiencies. Initial signs and symptoms can be nonspecific, contributing to late diagnosis. Loss of zona glomerulosa function may precede zona fasciculata and reticularis deficiencies. Patients present with hallmark manifestations including fatigue, weight loss, abdominal pain, melanoderma, hypotension, salt craving, hyponatremia, hyperkalemia, or acute adrenal crisis. Diagnosis is established by unequivocally low morning serum cortisol/aldosterone and elevated ACTH and renin concentrations. A standard dose $(250 \mu \mathrm{g})$ Cosyntropin stimulation test may be needed to confirm adrenal insufficiency (Al) in partial deficiencies. Glucocorticoid and mineralocorticoid substitution is the hallmark of treatment, alongside patient education regarding dose adjustments in periods of stress and prevention of acute adrenal crisis. Recent studies identified partial residual adrenocortical function in patients with $\mathrm{Al}$ and rare cases have recuperated normal hormonal function. Modulating therapies using rituximab or $\mathrm{ACTH}$ injections are in early stages of investigation hoping it could maintain glucocorticoid residual function and delay complete destruction of adrenal cortex.

Keywords: cortisol, aldosterone, ACTH, renin, autoimmunity, hypocortisolism, adrenal insufficiency

\section{DEFINITION AND EPIDEMIOLOGY}

The adrenal cortex produces glucocorticoids, mineralocorticoids and androgens, under the influence of adrenocorticotropic hormone (ACTH) and the renin-angiotensin system $(1,2)$. In the event of adrenal cortex destruction, primary adrenal insufficiency (PAI) develops and is characterized by reduced serum concentrations of all three hormones: cortisol, aldosterone and adrenal androgens (3). However, since intra-adrenal cortisol is required for epinephrine production by the adrenal medulla, PAI is often associated with decreased phenylethanolamine N-methyltransferase (PNMT) activity, resulting in adrenomedullary dysfunction $(1,4)$. Central adrenal insufficiency (AI), encompasses both secondary and tertiary AI caused by low ACTH and low corticotropin releasing hormone $(\mathrm{CRH})$, respectively (5). However, since aldosterone production is mainly controlled by renin, angiotensin II and potassium $(1,6)$, it is not affected in central AI. 
PAI was first described by Thomas Addison in 1855 in a case series of 11 patients and therefore it is often called Addison's disease (7). Since then, prevalence has been on the rise especially in Europe, reaching $117 /$ million in central Italy in the late 1990 s (8) and 144/million in Norway in 2007 (9). More recently, an even higher prevalence was documented in a 2016 Icelandic nationwide study of patients over 18 years of age: $221 /$ million population (10). Furthermore, an annual average increase in the prevalence of PAI of $1.8 \%$ per year was reported in Germany from 2008 to 2012 (11). The annual incidence of PAI is estimated to be around $0.44-0.62$ per $100,000(9,12)$. PAI is more frequently found in women than men $(11,13)$ with a M:F ratio of 1: 3.5 (14). Age of onset is typically around 30-50 years old $(1,9,10,14)$. Autoimmune destruction of adrenal cortex has surpassed tuberculosis as the most common cause of PAI, in particular in high income countries $(1,15)$. Comorbid autoimmune disorders can often be found in patients with autoimmune AI (AAI), reported to be 46.5 and $66 \%$, respectively in the German (11) and Norwegian studies (9). Most common comorbid conditions were thyroid disease, type 1 diabetes, vitiligo, vitamin B12 deficiency and primary ovarian insufficiency (POI) (9-11). When occurring with other autoimmune disorders, AAI may be part of an autoimmune polyglandular syndrome (APS). APS type 1 or autoimmune polyendocrinopathy-candidiasis-ectodermal dystrophy (APECED) is a rare monogenic disease, in which the autoimmune regulator gene (AIRE) is mutated and at least 2 of the following three occur: chronic mucocutaneous candidiasis, hypoparathyroidism and PAI (16). Generally, its transmission is autosomal recessive but may be dominant (16). Other conditions may include POI, enteropathy and rarely lymphomas $(6,16)$. More commonly found is APS type 2, a polygenic condition in which genes encoding cytotoxic T-lymphocyte-associated protein 4 (CTLA-4), protein tyrosine phosphatase, nonreceptor type 22 (PTPN22), the transcriptional regulator protein $\mathrm{BACH} 2$, and the CD25-interleukin-2 receptor have been implicated (16), along with associations to certain haplotypes including DR3DQ2, DR4-DQ8, DRB1-0301, and DRB1-0404 (15, 17). It commonly associates with type 1 diabetes and autoimmune thyroid disease (16). When AAI is confirmed alongside other autoimmune diseases, excluding the cardinal ones needed for diagnosis of APS types 1 and 2, APS type 4 is diagnosed (5).

\section{SIGNS AND SYMPTOMS OF AI- NATURAL HISTORY OF AUTOIMMUNE PAI-MORTALITY}

Onset of AI can be insidious, with many of the symptoms and signs being nonspecific and often leading to a delay in diagnosis (1). In a retrospective study including 216 patients with AI (18), more than half of patients consulted at least 3 physicians and were falsely diagnosed with either gastrointestinal or psychiatric disorders most frequently. A 5 year delay in diagnosis was reported in as much as $20 \%$ of patients (18). Symptoms of cortisol deficiency are common to both primary and central AI. These include loss of appetite, weight loss, lethargy, gastrointestinal symptoms such as nausea, abdominal pain and vomiting $(1,6)$.

Because aldosterone deficiency exists in PAI but not in central AI, symptoms and signs of mineralocorticoid deficiency including, dizziness and orthostatic hypotension, salt craving, hyponatremia, hyperkalemia and hyperchloremic acidosis, can be found $(1,19)$. Orthostatic hypotension may also be secondary to cortisol deficiency via reduced expression of catecholamine receptors on blood vessels and therefore may be found in central AI, albeit less pronounced than in PAI (1, 20). Although hyponatremia can be present in both primary and central AI, the underlying mechanisms differ. In PAI, hyponatremia is secondary to aldosterone deficiency resulting in renal salt wasting and hypovolemia with an increased risk of dehydration and acute kidney injury $(19,21)$. In central AI, it is a result of increased vasopressin secondary to cortisol deficiency, which in turn results in water retention and euvolemic hyponatremia (22). Other possible laboratory abnormalities are hypercalcemia, mild normocytic anemia, lymphocytosis, eosinophilia and hypoglycemia which occurred more frequently in children $(1,6)$. There is also a significant decrease in natural killer cells cytotoxicity in patients with PAI, compromising innate immunity, hence contributing to increased viral infections (23). Androgen deficiency may result in low libido or reduced energy as well as thinning of axillary and pubic hair in post-menopausal women $(1,3)$. In pregnant women with unrecognized AI, establishing the diagnosis based on signs and symptoms is challenging, because cortisol deficiency symptoms may also be seen as part of a normal pregnancy. While only a few cases were diagnosed during pregnancy, the high maternal and fetal risks associated with unrecognized AI should prompt physicians to suspect the diagnosis in women with symptoms persisting into the second trimester or occurring secondary to illness or labor (24).

A particularly distinctive feature of PAI is melanoderma which can be explained by elevated ACTH due to loss of negative feedback control usually exerted by cortisol on corticotropic cells in anterior pituitary gland (1). Elevated plasma ACTH activates melanocortin 1 receptors (M1R), resulting in excessive pigmentation, especially on areas exposed to sun and friction such as face, neck, knuckles, creases in the hand, elbows, areola of the nipple, scrotum, labia and newly acquired scars $(6,19)$. Mucosal pigmentation can also be noted and clinicians should look for brown patchy discoloration on lips, palate and gingiva (19).

PAI may be underrecognized because of the nonspecific symptoms of cortisol deficiency, especially in its early stages. This is often called latent AI and should be suspected in the presence of unexplained health complaints related to stress, such as gastrointestinal symptoms, fatigue and weight loss, in particular in patients with a history of autoimmune disease (25, 26). Betterle et al. suggested that autoimmune adrenocortical destruction goes through 4 stages (27) (Figure 1). The very first stage of the disease is marked by aldosterone deficiency 


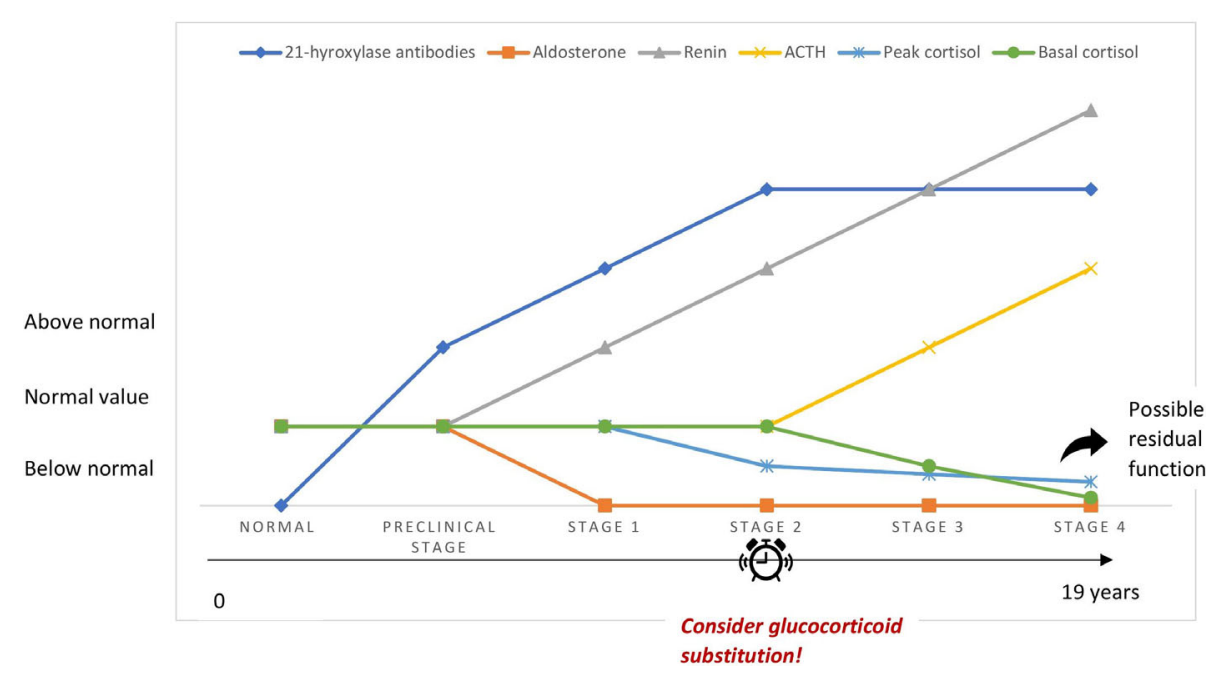

FIGURE 1 | Simplified schematic depiction of the 4 stages of autoimmune adrenocortical destruction.

and elevated renin concentrations suggesting that zona glomerulosa may be more prone to autoimmune destruction than zona fasciculata (27-29). The latter may be initially spared owing to the high local concentrations of cortisol responsible for suppressing antigen presentation to immune cells and procuring local anti-inflammatory properties (30). The second stage is characterized by an impaired cortisol response to synthetic ACTH administered intravenously, while in third and fourth stages, ACTH increases and serum cortisol drops, respectively (27). Early stages of AAI are difficult to diagnose because of the asymptomatic or pauci-symptomatic state since adrenal function is maintained by high plasma ACTH/renin (31). Stages 1 and 2 may be reversible in sporadic AAI. This was not true in APS types 1 and 2/4, where respectively stage 1 and stage 2 were defined as the point of no return with a cumulative risk of $100 \%$ (28). Progression of AAI occurred in most patients within 11 years, while no progression was seen after 19 years of follow up in a large cohort of 143 antibody positive patients. Progression was higher in males, patients who had APS type 1 and impaired adrenal function (28). However, antibody titers did not affect risk of progression, probably because AAI is mediated by cellular immunity and antibodies are in fact markers of the disease (27, 28). In 100 asymptomatic antibody positive patients followed up for 20 years, impairment of adrenal function occurred at an annual rate of $4.9 \%$ (29). Treatment with glucocorticoids in stressful conditions such as a major surgery or illness may be considered for patients at stages 1 and 2 and close monitoring of patients with higher risk of progression to clinical disease is warranted (children, APS type 1, high antibody titers and already at stage 1) $(29,32)$.

Because of the insidious course of the disease, patients may not be diagnosed until they present with adrenal crisis. Adrenal crisis is an acute, life-threatening condition more frequently seen in PAI than central AI (33). The frequency of adrenal crisis varied from 4.4 to $17 / 100$ patient-years $(11,34,35)$, depending on the definition used for adrenal crises and the characteristics of patients included in each of the studies. A prospective study by Hahner et al., evaluated the incidence of adrenal crisis in 423 patients followed up for 2 years. It was found to be persistently high even in adequately educated patients, reaching 8.3 crises/ 100 patient-years with a high mortality, estimated at 0.5 adrenal crisis related deaths/100 patient-years (36). A much lower incidence was reported in Switzerland despite insufficient patient education regarding their disease (37). Recently, an analysis of the biggest cohort described to date, including 2694 patients from the European Adrenal Insufficiency Registry, revealed an adrenal crisis incidence of $6.53 / 100$ patient-years, with more than one episode per year in $16 \%$ of patients with PAI (38). In the absence of a universal definition of adrenal crisis, its recognition can prove to be difficult. The most used definition is an unstable hemodynamic state (absolute or relative hypovolemia/shock) recovering within 1 to 2 hours following parenteral glucocorticoid administration (4, 33). Although hyponatremia and hyperkalemia are very common features in adrenal crisis, clinicians should beware of patients with multiple episodes of vomiting and severe dehydration because they can present with normal levels of serum potassium and sodium (6). Adrenal crisis can be triggered by numerous precipitating events, namely gastrointestinal infections, fever, emotional stress, major surgery, pregnancy, strenuous physical activity and noncompliance $(34-37,39)$. In $7-14 \%$ of adrenal crises, no precipitating event could be identified $(34,36)$. Patients at higher risk of adrenal crisis were especially those who have a history of a previous adrenal crisis (threefold increase) (36), but also patients that are female (35), older (40) and have diabetes insipidus (35), type 1 diabetes mellitus (41) and cardiac, neurological or pulmonary comorbidities $(35,39)$. Conversely, the latter comorbidities were not shown to be associated with a higher risk of adrenal crises according to the new study by Quinkler et al. (38). However, they did have more infections associated with adrenal crisis occurrence, confirming 
their immunocompromised state and inability to defend against viral infections owing to a natural killer cell dysfunction $(23,38)$. Eight \% of annual cases of adrenal crises will need in hospital treatment and admission rates were highest in patients aged more than 60 years old (41). In children, adrenal crisis frequency was reported to be 3.4/100 patient-years and children diagnosed with salt wasting congenital adrenal hyperplasia (CAH), adrenal hypoplasia congenital (AHC) and AAI were at higher risk (42). Compared to the general population, patients with AI often complain of reduced quality of life and work capacity with more sick day leaves potentially explained by the inability of present treatment modalities to replicate circadian rhythm of cortisol production $(6,13)$. Another significant issue is the impact of PAI on fertility in women of reproductive age. Few cohort studies have shown that pregnancy rate decreases after diagnosis of PAI even in the absence of associated POI, and a higher risk of cesarean delivery, impaired fetal growth, preterm birth and low birth weight is found (43). Particularly, a German study including 39 women with $\mathrm{CAH}$ secondary to 21-hydroxylase deficiency and 54 with AAI, reported reduced fertility only in classic CAH and APS type 2, based on answers from a self-reporting questionnaire (44). This suggests that a more severe course of disease and/or the presence of other comorbid autoimmune disorders largely impact fertility outcomes. There are no clear explanations for the underlying mechanisms of reduced fertility in women with AAI, in the absence of POI. Perhaps their reduced quality of life and overall dissatisfaction with current treatment modalities may play a significant role in hindering successful pregnancies.

\section{ETIOLOGIES}

The most common cause of PAI in the adult population is autoimmune destruction of the adrenal cortex (1). Other potential causes include infections, infiltrative diseases, adrenal hemorrhage, surgery, and drugs $(1,6)$. They are summarized in Table 1. AAI remains a diagnosis of exclusion and in the absence of positive antibodies directed against 21-hydroxylase, other diagnoses should be explored; an abdominal computed tomography should be performed. In the event of enlarged adrenal glands, differential diagnoses include an active tuberculous infection, systemic fungal infections in immunocompromised patients, metastases and lymphoma (1). In young men with negative antibodies to 21-hydroxylase, adrenoleukodystrophy should be suspected, even in the absence of neurological symptoms $(1,6)$. Adrenoleukodystrophy is an X-linked recessive disorder, hence affecting only boys, characterized by defects in $A B C D 1$ gene causing elevated serum very long chain fatty acids. The clinical spectrum of adrenoleukodystrophy includes cerebral manifestations that may manifest in childhood such as behavior changes, school difficulties, cognitive deficits up to dementia, psychoses and loss of vision and speech. It may be associated with adrenomyeloneuropathy presenting later in middle age with progressive lower body stiffness and weakness (45). The diagnosis of adrenoleukodystrophy should be confirmed by molecular genetic testing (46). In children, however, CAH represents $83 \%$ of PAI diagnoses compared to $9.7,6.1$ and $1.2 \%$ for AHC, autoimmune AI and adrenoleukodystrophy, respectively (42). $97.2 \%$ of CAH were secondary to 21 -hydroxylase deficiency in a Chinese pediatric cohort followed up for 29 years (47). A detailed discussion of these diagnoses is outside the scope of this review and will be discussed elsewhere.

\section{PATHOGENESIS OF AUTOIMMUNE AI}

AAI is an autoimmune destructive process affecting all three zones of the adrenal cortex in which lymphocytes infiltrate the adrenal parenchyma leading to adrenal fibrosis and atrophy. It is a slow process and AI may not be clinically relevant until most of adrenocortical cells are destroyed (48). Antibodies directed against 21-hydroxylase are specific to AAI and are rarely seen in the general population; hence their presence alongside positive adrenal cortex antibodies allow to accurately diagnose AAI in $99 \%$ of cases $(19,49,50)$. The presence of antibodies targeting interferon- $\alpha 2$ and interferon- $\omega$ should prompt genetic testing for APS type $1(19,49)$. While the detection of anti 21hydroxylase antibodies is diagnostic of AAI, they have no direct role in pathogenesis and are only biologic markers of autoimmunity $(48,49)$. In fact, cytotoxic T-cells auto-reactive to steroidogenic enzymes, in particular 21-hydroxylase, infiltrate the adrenal cortex in response to proinflammatory chemokines, CXCL9 and CXCL10, released intrinsically by adrenocortical cells. Hence, adrenocortical cells contribute to their own destruction in the presence of both, a genetic predisposition and environmental triggers (48). Possible triggers are thought to be caused by local viral infections with increased tropism to adrenocortical cells, such as herpes simplex virus 1, cytomegalovirus and adenovirus, as well as interferon alfa treatments and the relatively new checkpoint inhibitors targeting CTLA-4, programmed cell death protein 1 (PD-1), and PD-1 ligand (PD-L1), used in melanoma and lung cancer treatment (48). Genetic variants implicated in AAI development include the autosomal recessive mutation in AIRE gene commonly responsible for APS type 1 (50) but was also implicated in AAI independently of APS type 1 in a recent genome wide association study (51), which reported 2 new alterations in $A I R E$, the strongest one being p.R471C. It also described nine independent risk loci implicated in central immunological tolerance (51), in particular PTPN22, CTLA4, and $\mathrm{BACH} 2$ loci whose role in pathogenesis is already established $(50,51)$ and SH2B3 and SIGLEC5 (51). HLA class II genes also play a central role in predisposition to isolated AAI or APS type 2/4. While HLA DRB1-0301 and DRB1-040 are associated with AAI, it seems that DRB1-0403 is protective against development of Addison's disease (50). Other polymorphisms worth mentioning include the MHC class I chain-related gene A (MICA) allele 5.1, the CIITA (MHC class II transactivator), the master regulator of $\mathrm{MHC}$ class II expression, STAT4, PD-L1, and the vitamin D receptor (50). All susceptibility loci described to date are in genes involved in adaptive or innate immunity (52), particularly affecting 
TABLE 1 | Etiologies of primary adrenal insufficiency in adults (1, 5, 6, 45).

\begin{tabular}{|c|c|}
\hline Etiology & Associated clinical/biological and radiological features \\
\hline AUTOIMMUNE ADRENALITIS & Bilateral adrenal atrophy on computed tomography. \\
\hline ISOLATED & $\begin{array}{l}\text { Polygenic- positive } 21 \text {-hydroxylase antibodies } \pm \text { adrenal cortex antibodies/17-hydroxylase } \\
\text { antibodies/steroid side chain cleavage enzyme autoantibodies- other Al diseases associated. }\end{array}$ \\
\hline APS TYPE 1 & $\begin{array}{l}\text { Autosomal recessive AIRE mutation- positive interferon antibodies- chronic mucocutaneous } \\
\text { candidiasis, hypoparathyroidism, ectodermal dystrophy. }\end{array}$ \\
\hline APS TYPE 2 & Polygenic- Type 1 diabetes and autoimmune thyroid disease \\
\hline \multicolumn{2}{|l|}{ INFECTIONS } \\
\hline ACTIVE TUBERCULOUS INFECTION & Enlarged adrenal glands on computed tomography, calcifications may be seen. \\
\hline \multicolumn{2}{|l|}{ SYSTEMIC FUNGAL INFECTIONS } \\
\hline WATERHOUSE-FRIDERICHSEN & Altered mental state, hypotension, fever, acute adrenal crisis. \\
\hline \multicolumn{2}{|l|}{ SYNDROME } \\
\hline \multicolumn{2}{|l|}{ SEPTIC CHOC } \\
\hline \multicolumn{2}{|l|}{ OPPORTUNISTIC INFECTIONS IN } \\
\hline \multicolumn{2}{|l|}{ IMMUNOCOMPROMISED } \\
\hline INFILTRATIVE DISEASES & Enlarged adrenal glands on computed tomography. \\
\hline METASTASES FROM LUNG, BREAST, & Known primary cancer. \\
\hline \multicolumn{2}{|l|}{ OR KIDNEY CARCINOMAS } \\
\hline SARCOIDOSIS & $\begin{array}{l}\text { Other signs specific to infiltrative disease (mediastinal lymph nodes, chronic kidney disease, } \\
\text { hypoparathyroidism, diabetes mellitus...) }\end{array}$ \\
\hline \multicolumn{2}{|r|}{ 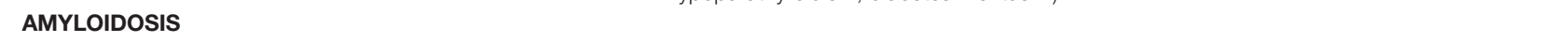 } \\
\hline HEMOCHROMATOSIS & Hypophysitis may be associated with infiltrative diseases. \\
\hline ADRENAL HEMORRHAGE & Sudden pain accompanied with acute adrenal crisis. \\
\hline COAGULATION DISORDERS & Adrenal hemorrhage on computed tomography. \\
\hline ANTICOAGULANT TREATMENT & Warfarin. \\
\hline ANTIPHOSPHOLIPID SYNDROME & Positive cardiolipin antibodies, lupus anticoagulant and anti-beta-2 glycoprotein 1. \\
\hline \multicolumn{2}{|l|}{ BILATERAL ADRENALECTOMY } \\
\hline PRIMARY ADRENAL LYMPHOMA & Enlarged adrenal glands on computed tomography. \\
\hline \multicolumn{2}{|l|}{ DRUGS } \\
\hline INCREASE CORTISOL METABOLISM & $\begin{array}{l}\text { Induction of P450-cytochrome enzymes, CYP3A4, CYP2B1, CYP2B2: phenytoin, rifampicin, } \\
\text { phenobarbital }\end{array}$ \\
\hline IMPAIRED STEROIDOGENESIS & $\begin{array}{l}\text { Ketoconazole, fluconazole, mitotane, metyrapone, etomidate, aminoglutethimide, trilostane. } \\
\text { Abiraterone acetate. }\end{array}$ \\
\hline ANTAGONIZE GLUCOCORTICOID ACTION & Mifepristone \\
\hline \multicolumn{2}{|l|}{ ON PERIPHERAL TISSUES } \\
\hline ADRENOLYTIC & Mitotane \\
\hline TRIGGER AUTOIMMUNE REACTION & Nivolumab and pembrolizumab \\
\hline \multirow[t]{6}{*}{ ADRENOLEUKODYSTROPHY } & Young men \\
\hline & X-linked recessive \\
\hline & Defects in $A B C D 1$ gene \\
\hline & Negative antibodies to 21-hydroxylase \\
\hline & Elevated serum very long chain fatty acids \\
\hline & Progressive neurological deficit, hypogonadism \\
\hline
\end{tabular}

regulatory $\mathrm{T}$-cell function leading to local intra-adrenal selfreactive cytotoxic T-cells (53). However, novel therapies targeting the cellular immune response or specific genes implicated in AAI pathogenesis have yet to emerge.

\section{ESTABLISHING THE DIAGNOSIS}

The Endocrine Society Clinical Practice Guideline published in 2016 recommend confirming the diagnosis of PAI with a corticotropin stimulation test to assess adrenocortical function (3). An algorithm depicting the proposed diagnostic approach in PAI in adults is shown in Figure 2. In PAI, adrenal glands are unresponsive to corticotropin stimulation because zona fasciculata is already maximally stimulated by elevated endogenous ACTH (54) and because adrenal cortex is replaced by fibrous tissue (48). In cases when confirmatory test is not possible, a morning serum cortisol of less than $140 \mathrm{nmol} / \mathrm{L}$ paired with a morning plasma ACTH above 2-fold the upper limit of normal is consistent with PAI. Measuring plasma renin and aldosterone is also recommended to document mineralocorticoid deficiency (3). There are two types of synthetic corticotropin analogs (ACTH 1-24) that can be used in the corticotropin stimulation test: cosyntropin (Cortrosyn, Amphastar Pharmaceuticals. Inc) and tetracosactrin (Synacthen, Novartis Pharma, Switzerland) and since they both exist in 250 $\mu \mathrm{g}$ formulations, the standard dose test is more practical and is recommended by the Endocrine Society Clinical Practice guideline (3). Diagnostic cutoff values of serum cortisol depend on the assay used: in most immunoassays, $500 \mathrm{nmol} / \mathrm{L}$ is often used as the cutoff to establish diagnosis (6). However, newer monoclonal immunoassays such as the Elecsys ${ }^{\circledR}$ Cortisol II from 


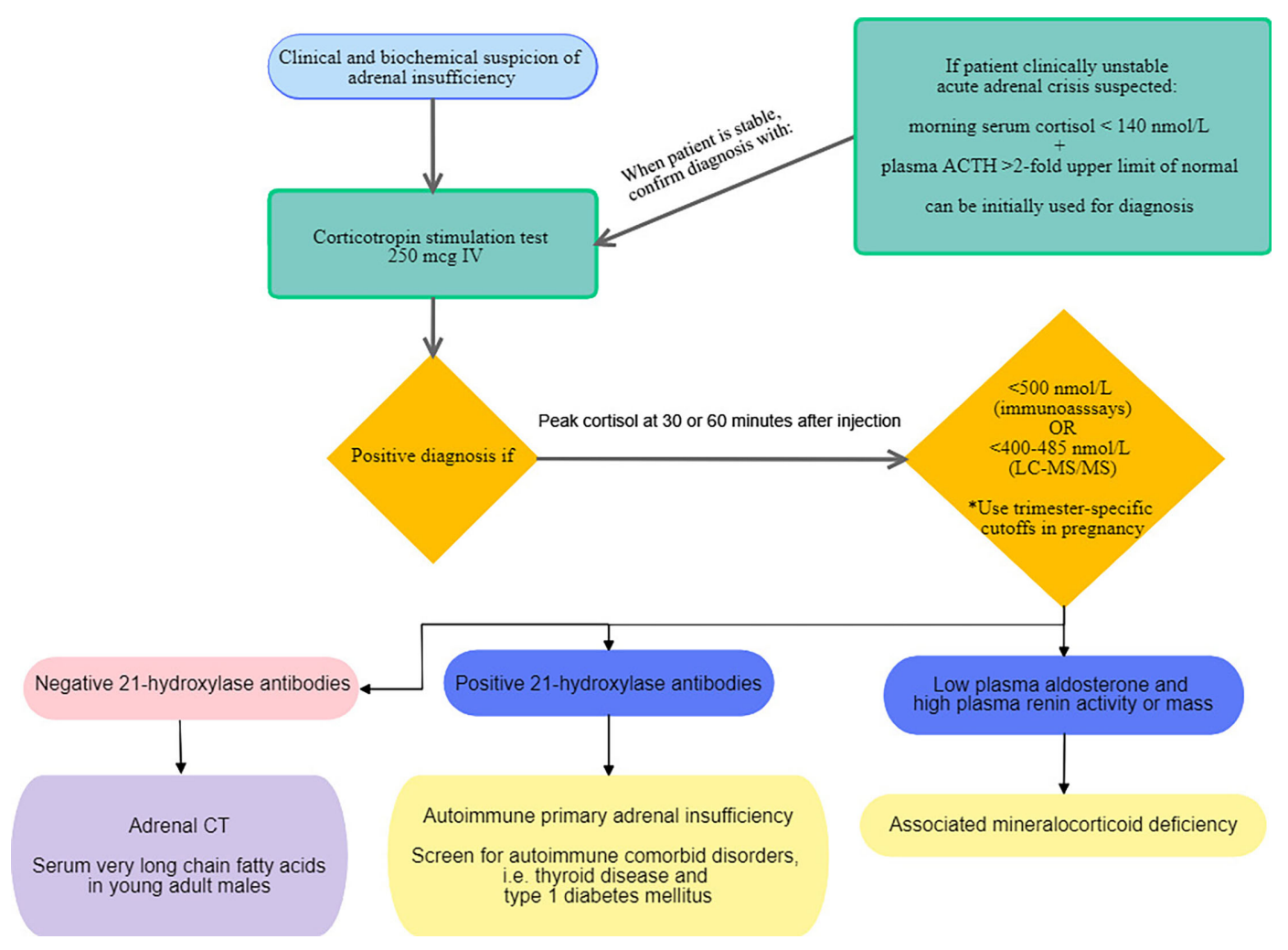

FIGURE 2 | Proposed diagnostic approach for primary adrenal insufficiency in adults.

Roche Diagnostics, have lower cross reactivity with other steroids, thus giving values that are $20-30 \%$ lower than those with older assays $(55,56)$. Two different studies suggested new cutoff thresholds when using the Elecsys ${ }^{\circledR}$ Cortisol II: $374 \mathrm{nmol} / \mathrm{L}$ (55) and $403 \mathrm{nmol} / \mathrm{L}$ at 30 minutes (56). Furthermore, because liquid chromatography tandem mass spectrometry (LC-MS/MS) measures cortisol more accurately than immunoassays, cutoff values were also redefined to be lower, respectively 400-412 and $485 \mathrm{nmol} / \mathrm{L}$, at 30 and 60 minutes following corticotropin analog injection $(56,57)$. This would potentially reduce overdiagnosis of AI. Additionally, a baseline cortisol less than $55 \mathrm{nmol} / \mathrm{L}$ successfully predicted abnormal response to stimulation test (56). A recent retrospective study conducted on 370 patients in Spain (58), confirmed need for sex-specific and assay-specific cutoff values when interpreting corticotropin test to reduce false positives and increase specificity. It also allowed for a better diagnostic agreement between sampling times at 30 and 60 minutes compared to using general cutoff values. Sampling at 30 minutes following injection of corticotropin analog accurately diagnosed $95 \%$ of PAI. However, this was not true for central AI, where sampling at 60 minutes showed better diagnostic accuracy (58). Corticotropin stimulation test can be done anytime of the day because in PAI response to ACTH is independent of circadian rhythm, but morning testing might be more accurate to avoid overdiagnosis in healthy individuals $(59,60)$. A metanalysis including 13 studies showed that the low dose corticotropin test $(1 \mu \mathrm{g})$ had better accuracy for diagnosing central AI compared to the standard dose $(250 \mu \mathrm{g})(61)$. Even so, the low dose is less practical because it necessitates dilution from supplied ampules of $250 \mu \mathrm{g}$ and is subject to human and technical errors $(3,61)$. Also, samples are best withdrawn 20 to 30 minutes following injection in order to avoid false positives $(59,61)$. Neither corticotropin stimulation test nor metyrapone test often used to diagnose central AI, can replace insulin tolerance test considered to be the gold standard for evaluation of hypothalamic pituitary axis. A study by Giordano et al. including 31 patients with central AI, failed to demonstrate superiority of either low dose or standard dose, when both achieved same diagnostic accuracy (62). Another potential role for low dose corticotropin test would be in establishing diagnosis of latent AAI. In fact, it was found abnormal in $88.4 \%$ of 33 patients with abnormal cortisol response compared to $66.6 \%$ for the standard dose, suggesting that the standard dose might miss some cases of latent PAI (63). More studies are needed to elucidate the diagnostic role of low dose corticotropin testing. Moreover, several factors may affect interpretation of cortisol response to stimulation tests. In particular, cortisol binding globulin $(\mathrm{CBG})$ which binds the majority of circulating cortisol, leaving only $5-10 \%$ of plasma cortisol free $(1,6)$, can be responsible for pitfalls in diagnosis. Because assays measure total serum cortisol and not free cortisol, conditions increasing CBG such as pregnancy, oral contraceptive pills and mitotane, 
lead to normal cortisol values, falsely reassuring clinicians $(6,64)$. Hence, trimester-specific cutoff values were suggested to eliminate false negatives: 700, 800 and $900 \mathrm{nmol} / \mathrm{L}$, respectively in first, second and third trimester (65). In situations where CBG is reduced (sepsis, cirrhosis, nephrotic syndrome, hyperthyroidism and SERPINA6 gene polymorphisms), low cortisol values must be interpreted with caution $(6,66)$. In such patients, measurement of salivary cortisol has the benefit of being a direct, noninvasive measurement of free cortisol and correlates well with circadian variations of serum cortisol (67). While it is more often used in the diagnosis of hypercortisolism, a recent study demonstrated an added benefit of measuring salivary cortisol in response to corticotropin stimulation in particular in patients taking oral estrogens and in cases of indeterminate serum cortisol at 60 minutes, defined as values between 500 and $599 \mathrm{nmol} / \mathrm{L}$ (68). The diagnostic cutoff used in this study was $26 \mathrm{nmol} / \mathrm{L}$ (68). Another emerging noninvasive diagnostic test is the measurement of salivary cortisol or cortisone at 60 minutes following administration of $500 \mu \mathrm{g}$ nasal tetracosactide with mucoadhesive chitosan. This generated the same 60 -minute plasma cortisol response as seen with $250 \mu \mathrm{g}$ intravenous tetracosactide and slightly lower levels of salivary cortisol and cortisone with the nasal formulation (69). Granted, this noninvasive diagnostic method is safe and convenient for patients, it requires additional studies before wide application can be recommended. Finally, measurement of anti 21-hydroxylase antibodies is necessary to establish autoimmune etiology. Commercially available assays include immunofluorescence and autoantibody assays. However, clinicians should keep in mind that they are not standardized and variations in between assays exist (3, 6). When AAI is diagnosed, screening for other comorbid autoimmune diseases should be undertaken; in particular autoimmune thyroid disease (70), type 1 diabetes (3) and POI, especially when steroid side chain cleavage enzyme autoantibodies are detected (29).

\section{MANAGEMENT OF CHRONIC AND ACUTE PAI}

Cortisol release follows a circadian and ultradian rhythm. It peaks early in the morning then gradually declines to reach nadir around midnight (6). Pulsatile release of cortisol every 60-90 minutes seems to be intrinsically related to interactions between the pituitary and the adrenal glands and might be independent of supra-pituitary influences (71). The Endocrine Society Clinical Practice Guideline recommends treating all patients with PAI with glucocorticoids and mineralocorticoids when aldosterone deficiency is confirmed (Figure 3). The authors agreed that hydrocortisone or cortisone acetate, given in two or three divided oral doses, should be the preferred therapeutic choices (3). Total daily dose should be the equivalent of 15-25 mg of hydrocortisone for adults, with the highest dose given in the morning, in an attempt to replicate the physiologic circadian rhythm (3). However, Caetano et al. recently showed that the daily hydrocortisone dose sufficient to substitute for glucocorticoid deficiency, without signs of under replacement, in 25 adults with AI, was significantly less than that recommended by the Endocrine Society Clinical Practice Guideline (72). The mean replacement dose reported in their study was $7.6 \pm 3.5 \mathrm{mg} / \mathrm{m} 2$, reflecting daily endogenous cortisol production (72). A recent systematic review of 47 studies reported that although prednisolone therapy increased risk of dyslipidemia and cardiovascular disease, it was as safe and efficacious as hydrocortisone (73). It also suggested that lower doses of hydrocortisone (less than $20 \mathrm{mg}$ /day) had better clinical outcomes, and failed to conclusively demonstrate an added benefit of modified release hydrocortisone or continuous subcutaneous hydrocortisone infusion using insulin pumps (73). Current available regimens fail to replicate both circadian and ultradian rhythmicity of cortisol, potentially explaining the persistently low quality of life that patients with AI often complain about. In fact, non-pulsatile cortisol secretion was found to be associated with poor quality of sleep, poor working memory performance and mood disorders $(74,75)$. An in-depth discussion of novel forms of glucocorticoid substitution and their role in better mimicking physiologic cortisol secretion will be covered in other chapters of this special topic.

Overreplacement of glucocorticoids predisposes to elevated blood pressure, diabetes, osteoporosis and obesity, and thus should be avoided (6). Monitoring of glucocorticoid therapy is based solely on clinical assessment using signs and symptoms of over and under replacement such as body weight, blood pressure, energy levels, hyperpigmentation and bone mineral density (3). Use of ACTH measurement is especially unreliable and will most definitely lead to overreplacement owing to the disrupted negative feedback of cortisol on ACTH (3). Although hair cortisol concentrations were found to be useful in identifying children overtreated with hydrocortisone, it requires a $1 \mathrm{~cm}$ thick hair sample and the technique is not yet widely available (76). Also, gene expression emerged recently as a potential tool for monitoring hydrocortisone therapy. In particular, expression of DSIPI, DDIT4 and FKBP5 increased 2 hours after hydrocortisone infusion and correlated well with normal serum cortisol levels (77).

Mineralocorticoid deficiency is treated with fludrocortisone, approximately 50-200 mcg in adults, in one single morning dose, and ad libitum salt consumption (3). Higher doses are often needed in specific circumstances such as in children because of early mineralocorticoid resistance, in athletic people and in very hot climates because of salt wasting due to excessive perspiration $(3,6)$. Clinicians should look for signs of overreplacement (hypertension, peripheral edema, hypokalemia) and those of under-replacement (orthostatic hypotension, salt craving and hyperkalemia). Adequate mineralocorticoid replacement can also be determined according to plasma renin concentration, which should be kept in the upper reference range (3).

Until this day, no evidence exists to support dehydroepiandrosterone (DHEA) replacement therapy in all patients with PAI. A trial therapy of 25-50 mg of DHEA for 


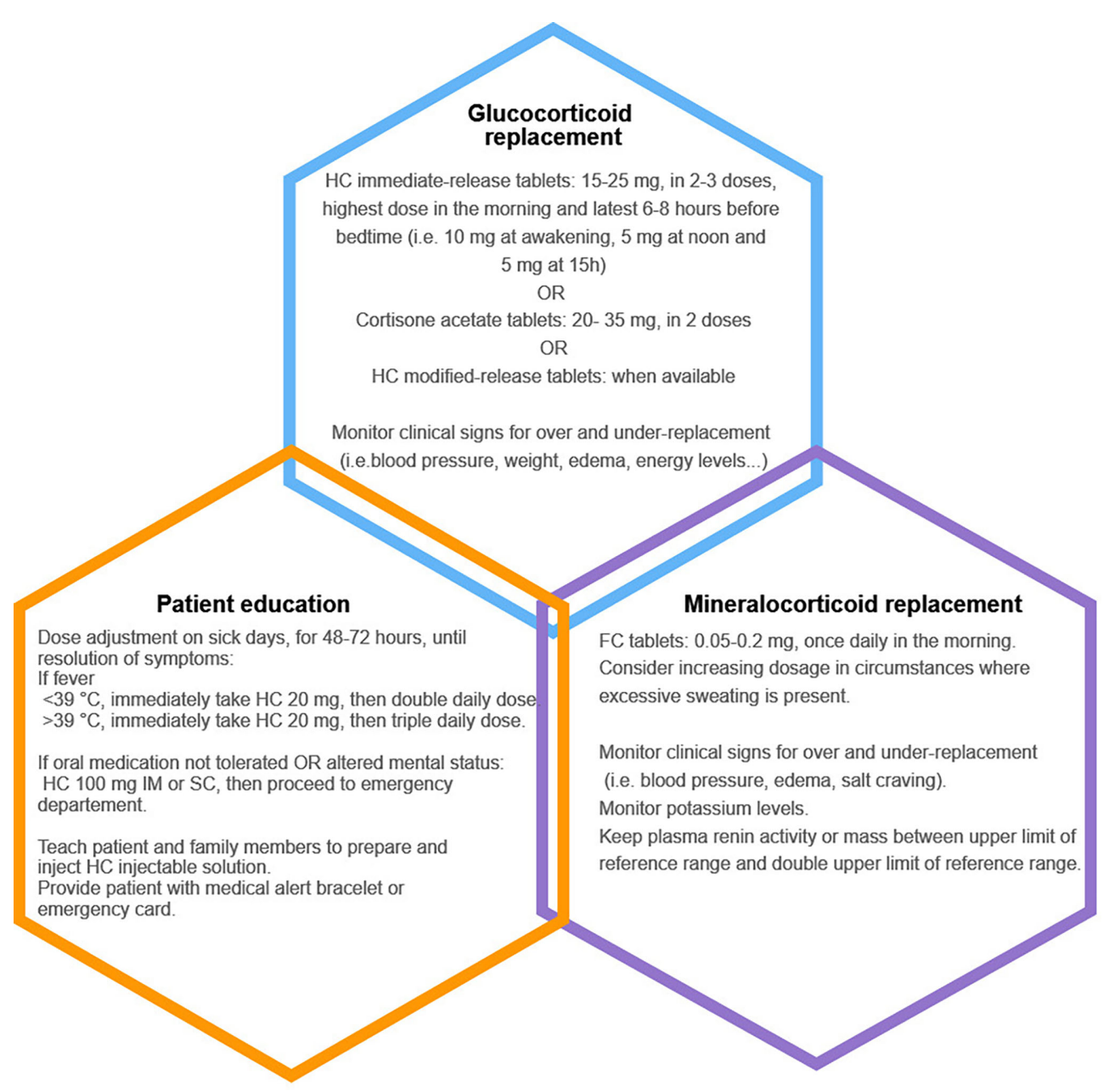

FIGURE 3 | Summary of the three pillars of management in adults with primary adrenal insufficiency. HC, hydrocortisone; FC, fludrocortisone; IM, intramuscularly; SC, subcutaneously.

6 months can be initiated in premenopausal women complaining of reduced libido and depressive moods despite adequate glucocorticoid and mineralocorticoid therapy $(3,6)$. Unfortunately, well standardized and reliable DHEA formulations are lacking in many countries.

Patient education on dose adaptation and sick day rules is an essential part of management (Figure 3). Because all patients with PAI are at risk of adrenal crisis, physicians should insist on increasing patient awareness to their disease by implementing treatment strategies, participating in group sessions, and prescribing at home hydrocortisone injection kits in case of emergencies (78). Hydrocortisone dose adjustments are required during sick days: patients should double or triple their daily oral dose for 48-72 hours until full recovery. If oral medication cannot be tolerated or absorbed, $100 \mathrm{mg}$ of parenteral hydrocortisone should be promptly administered before seeking medical care (3). Subcutaneous hydrocortisone injection is an alternative in patients who cannot self-inject hydrocortisone intramuscularly. It was shown that cortisol increased rapidly following subcutaneous hydrocortisone with a delay of only 11 minutes when compared to intramuscular hydrocortisone (79). A medical alert bracelet or an emergency card can help health care providers identify patients with PAI requiring lifesaving hydrocortisone administration in emergent situations (78). During adrenal crises, it is recommended to administer $100 \mathrm{mg}$ of intravenous hydrocortisone followed by either continuous infusion of $200 \mathrm{mg}$ of hydrocortisone per 24 hours or $50 \mathrm{mg}$ intravenously every 6 hours alongside adequate isotonic saline infusion. Tapering of hydrocortisone can begin 24-48 hours following adrenal crisis, when patients can tolerate oral medication. Fludrocortisone can be re-introduced when hydrocortisone dose is less than $50 \mathrm{mg} /$ day $(3,6)$. Stress dosing is also needed before dental and minor surgeries $(25-75 \mathrm{mg} / 24 \mathrm{~h}$ of hydrocortisone, depending on type of procedure). Major surgeries, trauma and delivery require same dosing regimen as in adrenal crises management (3). Management of PAI in special populations including children, patients with $\mathrm{CAH}$, pregnant women and endurance athletes is not discussed in this review. 


\section{RESIDUAL GLUCOCORTICOID FUNCTION AND FUTURE PERSPECTIVES}

Adrenocortical plasticity is a well-established concept defined by the ability of subcapsular adrenocortical stem cells to proliferate, then migrate into the three zones of the adrenal cortex where zone-specific differentiation occurs, and cells acquire steroidogenic function; all under the influence of ACTH (54). Adrenal mass is also influenced by ACTH: when deficient, adrenal atrophy and hypofunction develop, whereas when increased, adrenal hyperplasia and hyperfunction occur (54). Adrenocortical stem cells may hold the key to understanding the mechanisms of a new emerging concept in autoimmune adrenalitis; that of residual glucocorticoid function. Indeed, residual glucocorticoid production was described in $30-50 \%$ of patients years after AAI was diagnosed $(80,81)$, more commonly in men and in those with a more recent diagnosis. In contrast, residual mineralocorticoid production was only found in $13.5 \%$ of patients (80). It was hypothesized that the lack of expression by adrenocortical stem cells of 21-hydroxylase and other enzymes implicated in steroidogenesis, may protect adrenal cortex from complete autoimmune destruction by retaining the possibility of repopulation by intact stem cells (54). Also, turnover of adrenocortical stem cells differs according to sex in rodents and could possibly explain why endogenous residual function is more commonly observed in men (54). Elevated ACTH in PAI may also contribute to the stimulation of proliferation and differentiation of adrenocortical stem cells. However, once hydrocortisone replacement therapy is introduced, endogenous cortisol production declines alongside the ACTH decline and it is not known whether this is responsible for a more rapid loss of residual function or it is but the natural history of autoimmune adrenalitis that is responsible for the loss of function over time (82). Glucocorticoid precursors such as 11deoxycortisol, 11-deoxycorticosterone and corticosterone, are potential biomarkers of residual endogenous adrenal function because their concentrations correlated well with serum cortisol $(81,82)$. Although, some patients retained residual adrenal function, they were not protected from increased risk of adrenal crisis and quality of life was still significantly altered (80). Spontaneous recovery of endogenous adrenal function in AAI is rarely described in the literature, 7-16 years following diagnosis, with most cases being partial recovery (83-85). Recent

\section{REFERENCES}

1. Oelkers W. Adrenal Insufficiency. N Engl J Med (1996) 335(16):1206-12. doi: 10.1056/NEJM199610173351607

2. Pazderska A, Pearce SH. Adrenal Insufficiency - Recognition and Management. Clin Med Lond Engl (2017) 17(3):258-62. doi: 10.7861/ clinmedicine.17-3-258

3. Bornstein SR, Allolio B, Arlt W, Barthel A, Don-Wauchope A, Hammer GD, et al. Diagnosis and Treatment of Primary Adrenal Insufficiency: An Endocrine Society Clinical Practice Guideline. J Clin Endocrinol Metab (2016) 101(2):364-89. doi: 10.1210/jc.2015-1710

4. Dineen R, Thompson CJ, Sherlock M. Adrenal Crisis: Prevention and Management in Adult Patients. (2019). Available at: https://www.ncbi.nlm. nih.gov/pmc/articles/PMC6566489/ (Accessed 2021 Apr 19). studies suggested that treatment with immunomodulators could be a step forward to reverse autoimmune destruction and allow for regeneration of adrenal cortex. Two of 13 patients who were given subcutaneous tetracosactide for a period of 20 weeks had urine glucocorticoid metabolite in the median range of healthy individuals and peak serum cortisol concentrations above 400 $\mathrm{nmol} / \mathrm{L}$, allowing for cessation of glucocorticoid therapy (86). A more recent study evaluated the effect of dual therapy with rituximab and depot tetracosactide in 13 patients with AAI (87). Although dual therapy did not allow full recovery of adrenal function, as defined in this study by peak cortisol $>$ $550 \mathrm{nmol} / \mathrm{L}$ at week 48 of treatment, it showed that endogenous cortisol production, quantified by urine metabolites, was increased in $62 \%$ of patients (87). Much remains to be explored in regenerative therapies and their role in recovering adrenal function. More studies are needed to allow for a better treatment approach that will mimic physiologic production of cortisol in order to ameliorate quality of life and reduce the morbidity related to PAI.

\section{CONCLUSION}

AAI remains a life-threatening condition if not recognized early. Despite medical advances, adrenal crises still occur and quality of life of patients is largely impacted. Attention should be especially given to patients with latent AI, at early stages of adrenal destruction, to prevent adrenal crises from developing and going unnoticed. Impending novel therapies are being explored to determine the best approach to utilize residual adrenal cortisol production and to regenerate adrenocortical cells destroyed by the autoimmune process. Until this is a validated practice, the only effective treatment remains adequate glucocorticoid and mineralocorticoid replacement and patient and physician education.

\section{AUTHOR CONTRIBUTIONS}

NY, IB, and AL contributed to conception and design of this review. NY wrote the first draft of the manuscript. IB and AL wrote sections of the manuscript and revised the final draft. All authors contributed to the article and approved the submitted version.

5. Charmandari E, Nicolaides NC, Chrousos GP. Adrenal Insufficiency. Lancet Lond Engl (2014) 383(9935):2152-67. doi: 10.1016/S0140-6736(13) 61684-0

6. Husebye ES, Pearce SH, Krone NP, Kämpe O. Adrenal Insufficiency. Lancet Lond Engl (2021) 397(10274):613-29. doi: 10.1016/S0140-6736(21) 00136-7

7. On the Constitutional and Local Effects of Disease of the Supra-Renal Capsules/by Thomas Addison. [Internet]. Available at: https://wellcome collection.org/works/xsmzqpdw (Accessed 2021 Apr 17).

8. Laureti S, Vecchi L, Santeusanio F, Falorni A. Is the Prevalence of Addison's Disease Underestimated? J Clin Endocrinol Metab (1999) 84(5):1762. doi: 10.1210/jcem.84.5.5677-7

9. Erichsen MM, Løvås K, Skinningsrud B, Wolff AB, Undlien DE, Svartberg J, et al. Clinical, Immunological, and Genetic Features of Autoimmune Primary 
Adrenal Insufficiency: Observations From a Norwegian Registry. J Clin Endocrinol Metab (2009) 94(12):4882-90. doi: 10.1210/jc.2009-1368

10. Olafsson AS, Sigurjonsdottir HA. Increasing Prevalence of Addison Disease: Results From a Nationwide Study. Endocr Pract Off J Am Coll Endocrinol Am Assoc Clin Endocrinol (2016) 22(1):30-5. doi: 10.4158/EP15754.OR

11. Meyer G, Neumann K, Badenhoop K, Linder R. Increasing Prevalence of Addison's Disease in German Females: Health Insurance Data 2008-2012. Eur $J$ Endocrinol (2014) 170(3):367-73. doi: 10.1530/EJE-13-0756

12. Løvås K, Husebye ES. High Prevalence and Increasing Incidence of Addison's Disease in Western Norway. Clin Endocrinol (Oxf) (2002) 56(6):787-91. doi: 10.1046/j.1365-2265.2002.t01-1-01552.x

13. Chabre O, Goichot B, Zenaty D, Bertherat JGroup 1. Epidemiology of Primary and Secondary Adrenal Insufficiency: Prevalence and Incidence, Acute Adrenal Insufficiency, Long-Term Morbidity and Mortality. Ann Endocrinol (2017) 78(6):490-4. doi: 10.1016/j.ando.2017.10.010

14. Kong MF, Jeffcoate W. Eighty-Six Cases of Addison's Disease. Clin Endocrinol (Oxf) (1994) 41(6):757-61. doi: 10.1111/j.1365-2265.1994.tb02790.x

15. Bancos I, Hahner S, Tomlinson J, Arlt W. Diagnosis and Management of Adrenal Insufficiency. Lancet Diabetes Endocrinol (2015) 3(3):216-26. doi: 10.1016/S2213-8587(14)70142-1

16. Husebye ES, Anderson MS, Kämpe O. Autoimmune Polyendocrine Syndromes. N Engl J Med (2018) 378(12):1132-41. doi: 10.1056/ NEJMra1713301

17. Dittmar M, Kahaly GJ. Polyglandular Autoimmune Syndromes: Immunogenetics and Long-Term Follow-Up. J Clin Endocrinol Metab (2003) 88(7):2983-92. doi: 10.1210/jc.2002-021845

18. Bleicken B, Hahner S, Ventz M, Quinkler M. Delayed Diagnosis of Adrenal Insufficiency Is Common: A Cross-Sectional Study in 216 Patients. Am J Med Sci (2010) 339(6):525-31. doi: 10.1097/MAJ.0b013e3181db6b7a

19. Saverino S, Falorni A. Autoimmune Addison's Disease. Best Pract Res Clin Endocrinol Metab (2020) 34(1):101379. doi: 10.1016/j.beem.2020.101379

20. Walker BR, Connacher AA, Webb DJ, Edwards CR. Glucocorticoids and Blood Pressure: A Role for the Cortisol/Cortisone Shuttle in the Control of Vascular Tone in Man. Clin Sci Lond Engl 1979 (1992) 83(2):171-8. doi: $10.1042 / \mathrm{cs} 0830171$

21. Arai K, Papadopoulou-Marketou N, Chrousos GP. Aldosterone Deficiency and Resistance. In: Endotext [Internet] (2000). South Dartmouth (MA: MDText.com, Inc. Available at: http://www.ncbi.nlm.nih.gov/books/ NBK279079/ (Accessed 2021 Apr 19).

22. Oelkers W. Hyponatremia and Inappropriate Secretion of Vasopressin (Antidiuretic Hormone) in Patients With Hypopituitarism. N Engl J Med (1989) 321(8):492-6. doi: 10.1056/NEJM198908243210802

23. Bancos I, Hazeldine J, Chortis V, Hampson P, Taylor AE, Lord JM, et al. Primary Adrenal Insufficiency Is Associated With Impaired Natural Killer Cell Function: A Potential Link to Increased Mortality. Eur J Endocrinol (2017) 176(4):471-80. doi: 10.1530/EJE-16-0969

24. Yuen KCJ, Chong LE, Koch CA. Adrenal Insufficiency in Pregnancy: Challenging Issues in Diagnosis and Management. Endocrine (2013) 44 (2):283-92. doi: 10.1007/s12020-013-9893-2

25. Yamamoto T. Latent Adrenal Insufficiency: Concept, Clues to Detection, and Diagnosis. Endocr Pract Off J Am Coll Endocrinol Am Assoc Clin Endocrinol (2018) 24(8):746-55. doi: 10.4158/EP-2018-0114

26. Yamamoto T. History of Stress-Related Health Changes: A Cue to Pursue a Diagnosis of Latent Primary Adrenal Insufficiency. Intern Med Tokyo Jpn (2014) 53(3):183-8. doi: 10.2169/internalmedicine.53.1156

27. Betterle C, Scalici C, Presotto F, Pedini B, Moro L, Rigon F, et al. The Natural History of Adrenal Function in Autoimmune Patients With Adrenal Autoantibodies. J Endocrinol (1988) 117(3):467-75. doi: 10.1677/ joe.0.1170467

28. Naletto L, Frigo AC, Ceccato F, Sabbadin C, Scarpa R, Presotto F, et al. The Natural History of Autoimmune Addison's Disease From the Detection of Autoantibodies to Development of the Disease: A Long-Term Follow-Up Study on 143 Patients. Eur J Endocrinol (2019) 180(3):223-34. doi: 10.1530/ EJE-18-0313

29. Betterle C, Coco G, Zanchetta R. Adrenal Cortex Autoantibodies in Subjects With Normal Adrenal Function. Best Pract Res Clin Endocrinol Metab (2005) 19(1):85-99. doi: 10.1016/j.beem.2004.11.008
30. Gan EH, Pearce SH. Management of Endocrine Disease: Regenerative Therapies in Autoimmune Addison's Disease. Eur J Endocrinol (2017) 176 (3):R123-35. doi: 10.1530/EJE-16-0581

31. Ketchum CH, Riley WJ, Maclaren NK. Adrenal Dysfunction in Asymptomatic Patients With Adrenocortical Autoantibodies. J Clin Endocrinol Metab (1984) 58(6):1166-70. doi: 10.1210/jcem-58-6-1166

32. Coco G, Dal Pra C, Presotto F, Albergoni MP, Canova C, Pedini B, et al. Estimated Risk for Developing Autoimmune Addison's Disease in Patients With Adrenal Cortex Autoantibodies. J Clin Endocrinol Metab (2006) 91 (5):1637-45. doi: 10.1210/jc.2005-0860

33. Rushworth RL, Torpy DJ, Falhammar H. Adrenal Crisis(2019). Available at: https://www.nejm.org/doi/10.1056/NEJMra1807486 (Accessed 2021 Apr 19).

34. Reisch N, Willige M, Kohn D, Schwarz H-P, Allolio B, Reincke M, et al. Frequency and Causes of Adrenal Crises Over Lifetime in Patients With 21Hydroxylase Deficiency. Eur J Endocrinol (2012) 167(1):35-42. doi: 10.1530/ EJE-12-0161

35. Hahner S, Loeffler M, Bleicken B, Drechsler C, Milovanovic D, Fassnacht M, et al. Epidemiology of Adrenal Crisis in Chronic Adrenal Insufficiency: The Need for New Prevention Strategies. Eur J Endocrinol (2010) 162(3):597-602. doi: 10.1530/EJE-09-0884

36. Hahner S, Spinnler C, Fassnacht M, Burger-Stritt S, Lang K, Milovanovic D, et al. High Incidence of Adrenal Crisis in Educated Patients With Chronic Adrenal Insufficiency: A Prospective Study. J Clin Endocrinol Metab (2015) 100(2):407-16. doi: 10.1210/jc.2014-3191

37. Notter A, Jenni S, Christ E. Evaluation of the Frequency of Adrenal Crises and Preventive Measures in Patients With Primary and Secondary Adrenal Insufficiency in Switzerland. Swiss Med Wkly (2018) 148:w14586. doi: 10.4414/smw.2018.14586

38. Quinkler M, Murray RD, Zhang P, Marelli C, Petermann R, Isidori AM, et al. Characterization of Patients With Adrenal Insufficiency and Frequent Adrenal Crises. Eur J Endocrinol (2021) 184(6):761-71. doi: 10.1530/EJE20-1324

39. Smans LCCJ, van der Valk ES, Hermus ARMM, Zelissen PMJ. Incidence of Adrenal Crisis in Patients With Adrenal Insufficiency. Clin Endocrinol (Oxf) (2016) 84(1):17-22. doi: 10.1111/cen.12865

40. Rushworth RL, Torpy DJ. A Descriptive Study of Adrenal Crises in Adults With Adrenal Insufficiency: Increased Risk With Age and in Those With Bacterial Infections. BMC Endocr Disord (2014) 14:79. doi: 10.1186/14726823-14-79

41. White K, Arlt W. Adrenal Crisis in Treated Addison's Disease: A Predictable But Under-Managed Event. Eur J Endocrinol (2010) 162(1):115-20. doi: 10.1530/EJE-09-0559

42. Eyal O, Levin Y, Oren A, Zung A, Rachmiel M, Landau Z, et al. Adrenal Crises in Children With Adrenal Insufficiency: Epidemiology and Risk Factors. Eur J Pediatr (2019) 178(5):731-8. doi: 10.1007/s00431-019-03348-1

43. Bensing S, Giordano R, Falorni A. Fertility and Pregnancy in Women With Primary Adrenal Insufficiency. Endocrine (2020) 70(2):211-7. doi: 10.1007/ s12020-020-02343-Z

44. Remde H, Zopf K, Schwander J, Quinkler M. Fertility and Pregnancy in Primary Adrenal Insufficiency in Germany. Horm Metab Res Horm Stoffwechselforschung Horm Metab (2016) 48(5):306-11. doi: 10.1055/s0035-1565183

45. Raymond GV, Moser AB, Fatemi A. X-Linked Adrenoleukodystrophy, in: (1993). Seattle (WA): University of Washington, Seattle: GeneReviews ${ }^{\circledR}$ [Internet]. Available at: http://www.ncbi.nlm.nih.gov/books/NBK1315/ (Accessed 2021 Apr 26).

46. Engelen M, Kemp S, de Visser M, van Geel BM, Wanders RJA, Aubourg P, et al. X-Linked Adrenoleukodystrophy (X-ALD): Clinical Presentation and Guidelines for Diagnosis, Follow-Up and Management. Orphanet J Rare Dis (2012) 7:51. doi: 10.1186/1750-1172-7-51

47. Wijaya M, Huamei M, Jun Z, Du M, Li Y, Chen Q, et al. Etiology of Primary Adrenal Insufficiency in Children: A 29-Year Single-Center Experience. J Pediatr Endocrinol Metab JPEM (2019) 32(6):615-22. doi: 10.1515/jpem2018-0445

48. Hellesen A, Bratland E, Husebye ES. Autoimmune Addison's Disease - An Update on Pathogenesis. Ann Endocrinol (2018) 79(3):157-63. doi: 10.1016/ j.ando.2018.03.008 
49. Husebye E, Løvås K. Pathogenesis of Primary Adrenal Insufficiency. Best Pract Res Clin Endocrinol Metab (2009) 23(2):147-57. doi: 10.1016/j.beem. 2008.09.004

50. Falorni A, Brozzetti A, Perniola R. From Genetic Predisposition to Molecular Mechanisms of Autoimmune Primary Adrenal Insufficiency. Front Horm Res (2016) 46:115-32. doi: 10.1159/000443871

51. Eriksson D, Røyrvik EC, Aranda-Guillén M, Berger AH, Landegren N, Artaza $\mathrm{H}$, et al. GWAS for Autoimmune Addison's Disease Identifies Multiple Risk Loci and Highlights AIRE in Disease Susceptibility. Nat Commun (2021) 12 (1):959. doi: 10.1038/s41467-021-21015-8

52. Mitchell AL, Pearce SHS. Autoimmune Addison Disease: Pathophysiology and Genetic Complexity. Nat Rev Endocrinol (2012) 8(5):306-16. doi: 10.1038/nrendo.2011.245

53. Betterle C, Presotto F, Furmaniak J. Epidemiology, Pathogenesis, and Diagnosis of Addison's Disease in Adults. J Endocrinol Invest (2019) 42 (12):1407-33. doi: 10.1007/s40618-019-01079-6

54. Pearce SHS, Gan EH, Napier C. Management of Endocrine Disease: Residual Adrenal Function in Addison's Disease. Eur J Endocrinol (2021) 184(2):R617. doi: 10.1530/EJE-20-0894

55. Raverot V, Richet C, Morel Y, Raverot G, Borson-Chazot F. Establishment of Revised Diagnostic Cut-Offs for Adrenal Laboratory Investigation Using the New Roche Diagnostics Elecsys ${ }^{\circledR}$ Cortisol II Assay. Ann Endocrinol (2016) 77 (5):620-2. doi: 10.1016/j.ando.2016.05.002

56. Javorsky BR, Raff H, Carroll TB, Algeciras-Schimnich A, Singh RJ, ColónFranco JM, et al. New Cutoffs for the Biochemical Diagnosis of Adrenal Insufficiency After ACTH Stimulation Using Specific Cortisol Assays. $J$ Endocr Soc (2021) 5(4):bvab022. doi: 10.1210/jendso/bvab022

57. Ueland GÅ, Methlie P, Øksnes M, Thordarson HB, Sagen J, Kellmann R, et al. The Short Cosyntropin Test Revisited: New Normal Reference Range Using Lc-Ms/Ms. J Clin Endocrinol Metab (2018) 103(4):1696-703. doi: 10.1210/ jc.2017-02602

58. Ortiz-Flores AE, Santacruz E, Jiménez-Mendiguchia L, García-Cano A, Nattero-Chávez L, Escobar-Morreale HF, et al. Role of Sampling Times and Serum Cortisol Cut-Off Concentrations on the Routine Assessment of Adrenal Function Using the Standard Cosyntropin Test in an Academic Hospital From Spain: A Retrospective Chart Review. (2018). Available at: https://www.ncbi. nlm.nih.gov/pmc/articles/PMC5942445/ (Accessed 2021 Apr 29).

59. Park YJ, Park KS, Kim JH, Shin CS, Kim SY, Lee HK. Reproducibility of the Cortisol Response to Stimulation With the Low Dose (1 Microg) of ACTH. Clin Endocrinol (Oxf) (1999) 51(2):153-8. doi: 10.1046/j.1365-2265.1999. 00773.x

60. Dickstein G, Shechner C, Nicholson WE, Rosner I, Shen-Orr Z, Adawi F, et al. Adrenocorticotropin Stimulation Test: Effects of Basal Cortisol Level, Time of Day, and Suggested New Sensitive Low Dose Test. J Clin Endocrinol Metab (1991) 72(4):773-8. doi: 10.1210/jcem-72-4-773

61. Kazlauskaite R, Evans AT, Villabona CV, Abdu TAM, Ambrosi B, Atkinson $\mathrm{AB}$, et al. Corticotropin Tests for Hypothalamic-Pituitary- Adrenal Insufficiency: A Metaanalysis. J Clin Endocrinol Metab (2008) 93(11):424553. doi: 10.1210/jc.2008-0710

62. Giordano R, Picu A, Bonelli L, Balbo M, Berardelli R, Marinazzo E, et al. Hypothalamus-Pituitary-Adrenal Axis Evaluation in Patients With Hypothalamo-Pituitary Disorders: Comparison of Different Provocative Tests. Clin Endocrinol (Oxf) (2008) 68(6):935-41. doi: 10.1111/j.13652265.2007.03141.x

63. Dekkers OM, Timmermans JM, Smit JWA, Romijn JA, Pereira AM. Comparison of the Cortisol Responses to Testing With Two Doses of ACTH in Patients With Suspected Adrenal Insufficiency. Eur J Endocrinol (2011) 164(1):83-7. doi: 10.1530/EJE-10-0621

64. van Seters AP, Moolenaar AJ. Mitotane Increases the Blood Levels of Hormone-Binding Proteins. Acta Endocrinol (Copenh) (1991) 124(5):52633. doi: 10.1530 /acta. 0.1240526

65. Lebbe M, Arlt W. What Is the Best Diagnostic and Therapeutic Management Strategy for an Addison Patient During Pregnancy? Clin Endocrinol (Oxf) (2013) 78(4):497-502. doi: 10.1111/cen.12097

66. Wallace I, Cunningham S, Lindsay J. The Diagnosis and Investigation of Adrenal Insufficiency in Adults. Ann Clin Biochem (2009) 46(5):351-67. doi: 10.1258/acb.2009.009101
67. Inder WJ, Dimeski G, Russell A. Measurement of Salivary Cortisol in 2012 Laboratory Techniques and Clinical Indications. Clin Endocrinol (Oxf) (2012) 77(5):645-51. doi: 10.1111/j.1365-2265.2012.04508.x

68. Nolan BJ, Sorbello J, Brown N, Dimeski G, Inder WJ. Characterization of the Serum and Salivary Cortisol Response to the Intravenous $250 \mu \mathrm{g}$ ACTH1-24 Stimulation Test. Endocrine (2018) 59(3):520-8. doi: 10.1007/s12020-017$1505-0$

69. Elder CJ, Vilela R, Johnson TN, Taylor RN, Kemp EH, Keevil BG, et al. Pharmacodynamic Studies of Nasal Tetracosactide With Salivary Glucocorticoids for a Noninvasive Short Synacthen Test. J Clin Endocrinol Metab (2020) 105(8):2692-703. doi: 10.1210/clinem/dgaa323

70. Yamamoto T. Comorbid Latent Adrenal Insufficiency With Autoimmune Thyroid Disease. Eur Thyroid J (2015) 4(3):201-6. doi: 10.1159/000433532

71. Lightman SL, Conway-Campbell BL. The Crucial Role of Pulsatile Activity of the HPA Axis for Continuous Dynamic Equilibration. Nat Rev Neurosci (2010) 11(10):710-8. doi: 10.1038/nrn2914

72. Caetano CM, Sliwinska A, Madhavan P, Grady J, Malchoff CD. Empiric Determination of the Daily Glucocorticoid Replacement Dose in Adrenal Insufficiency. J Endocr Soc (2020) 4(11):bvaa145. doi: 10.1210/ jendso/bvaa145

73. Kiko N, Kalhan A. Comparison of Various Glucocorticoid Replacement Regimens Used in Chronic Adrenal Insufficiency: A Systematic Review. Dubai Diabetes Endocrinol J (2020) 26(2):50-68. doi: 10.1159/000508321

74. Kalafatakis K, Russell GM, Harmer CJ, Munafo MR, Marchant N, Wilson A, et al. Ultradian Rhythmicity of Plasma Cortisol Is Necessary for Normal Emotional and Cognitive Responses in Man. Proc Natl Acad Sci (2018) 115 (17):E4091-100. doi: 10.1073/pnas.1714239115

75. Kalafatakis K, Russell GM, Lightman SL. Mechanisms in Endocrinology: Does Circadian and Ultradian Glucocorticoid Exposure Affect the Brain? Eur J Endocrinol (2019) 180(2):R73-89. doi: 10.1530/EJE-18-0853

76. Noppe G, van Rossum EFC, Vliegenthart J, Koper JW, van den Akker ELT. Elevated Hair Cortisol Concentrations in Children With Adrenal Insufficiency on Hydrocortisone Replacement Therapy. Clin Endocrinol (Oxf) (2014) 81 (6):820-5. doi: 10.1111/cen.12551

77. Sævik ÅB, Wolff AB, Björnsdottir S, Simunkova K, Hynne MS, Dolan DWP, et al. Potential Transcriptional Biomarkers to Guide Glucocorticoid Replacement in Autoimmune Addison's Disease. J Endocr Soc (2021) 5(3): bvaa202. doi: 10.1210/jendso/bvaa202

78. Guignat L. Therapeutic Patient Education in Adrenal Insufficiency. Ann Endocrinol (2018) 79(3):167-73. doi: 10.1016/j.ando.2018.03.002

79. Hahner S, Burger-Stritt S, Allolio B. Subcutaneous Hydrocortisone Administration for Emergency Use in Adrenal Insufficiency. Eur J Endocrinol (2013) 169(2):147-54. doi: 10.1530/EJE-12-1057

80. Sævik ÅB, Åkerman A-K, Methlie P, Quinkler M, Jørgensen AP, Höybye C, et al. Residual Corticosteroid Production in Autoimmune Addison Disease. J Clin Endocrinol Metab (2020) 105(7):2430-41. doi: 10.1210/ clinem/dgaa256.

81. Vulto A, Bergthorsdottir R, van Faassen M, Kema IP, Johannsson G, van Beek AP. Residual Endogenous Corticosteroid Production in Patients With Adrenal Insufficiency. Clin Endocrinol (Oxf) (2019) 91(3):383-90. doi: $10.1111 /$ cen.14006

82. Napier C, Allinson K, Gan EH, Mitchell AL, Gilligan LC, Taylor AE, et al. Natural History of Adrenal Steroidogenesis in Autoimmune Addison's Disease Following Diagnosis and Treatment. J Clin Endocrinol Metab (2020) 105(7):2322-30. doi: 10.1210/clinem/dgaa 187

83. Smans LCCJ, Zelissen PMJ. Partial Recovery of Adrenal Function in a Patient With Autoimmune Addison's Disease. J Endocrinol Invest (2008) 31(7):672-4. doi: $10.1007 / \mathrm{BF} 03345623$

84. Baxter M, Gorick S, Swords FM. Recovery of Adrenal Function in a Patient With Confirmed Addison's Disease. Endocrinol Diabetes Metab Case Rep (2013) 2013:130070. doi: 10.1530/EDM-13-0070

85. Nordin BE. Addison's Disease With Partial Recovery. Proc R Soc Med (1955) 48(12):1024-6.

86. Gan EH, MacArthur K, Mitchell AL, Hughes BA, Perros P, Ball SG, et al. Residual Adrenal Function in Autoimmune Addison's Disease: Improvement After Tetracosactide (ACTH1-24) Treatment. J Clin Endocrinol Metab (2014) 99(1):111-8. doi: 10.1210/jc.2013-2449 
87. Napier C, Gan EH, Mitchell AL, Gilligan LC, Rees DA, Moran C, et al. Residual Adrenal Function in Autoimmune Addison's Disease-Effect of Dual Therapy With Rituximab and Depot Tetracosactide. J Clin Endocrinol Metab (2020) 105(4):e1250-9. doi: 10.1210/clinem/dgz287

Conflict of Interest: The authors declare that the research was conducted in the absence of any commercial or financial relationships that could be construed as a potential conflict of interest.

Publisher's Note: All claims expressed in this article are solely those of the authors and do not necessarily represent those of their affiliated organizations, or those of the publisher, the editors and the reviewers. Any product that may be evaluated in this article, or claim that may be made by its manufacturer, is not guaranteed or endorsed by the publisher.

Copyright $\odot 2021$ Younes, Bourdeau and Lacroix. This is an open-access article distributed under the terms of the Creative Commons Attribution License (CC BY). The use, distribution or reproduction in other forums is permitted, provided the original author(s) and the copyright owner(s) are credited and that the original publication in this journal is cited, in accordance with accepted academic practice. No use, distribution or reproduction is permitted which does not comply with these terms. 